\title{
THE GRAVITATIONAL DEMISE OF COLD DEGENERATE STARS
}

\author{
Fred C. Adams, Gregory Laughlin, and Manasse Mbonye \\ Physics Dept., University of Michigan, Ann Arbor, MI 48109, USA \\ Malcolm J. Perry \\ DAMTP, University of Cambridge, Silver Street, Cambridge, CB3 9EW, England \\ 19 May 1998
}

\begin{abstract}
We consider the long term fate and evolution of cold degenerate stars under the action of gravity alone. Although such stars cannot emit radiation through the Hawking mechanism, the wave function of the star will contain a small admixture of black hole states. These black hole states will emit radiation and hence the star can lose its mass energy in the long term. We discuss the allowed range of possible degenerate stellar evolution within this framework.
\end{abstract}

PACS Numbers: 97.60.Jd,Lf,Sm; 4.40.Dg; 4.70.Dy 


\section{INTRODUCTION}

White dwarfs are usually considered to be the final state of stellar evolution for nearly all stars in the universe [1]. The remaining stars are destined to end their lives as neutron stars or black holes. A fundamental astrophysical question is to determine the long term fate and evolution of these stellar remnants. If the proton is unstable through some process at the unification scale $M_{X}$ [2], then long term stellar evolution will be governed by the physics of proton decay with a time scale of

$$
\tau_{P} \sim 10^{32} \mathrm{yr}\left(M_{X} / 10^{15} \mathrm{GeV}\right)^{4} .
$$

This evolutionary path has been studied previously $[1,3,4]$. In this paper, we consider the ultimate fate of these cold degenerate stars in the absence of unification scale proton decay, i.e., due to the influence of only the gravitational force. In particular, we consider processes involving black holes and their effects on the long term evolution of these degenerate stars.

If the proton were truly stable, then nature presents us with a curious state of affairs. Black holes of stellar mass, which are much more tightly bound than degenerate stars, will evaporate through the Hawking effect [5] with a lifetime of only $\sim 10^{66} \mathrm{yr}[6-8]$. Although this time scale seems long compared to the current age of the universe $\left(\sim 10^{10} \mathrm{yr}\right)$, it is vastly shorter than the expected lifetimes of white dwarfs and neutron stars. These stellar remnants are essentially zero temperature objects; they exist in hydrostatic equilibrium due to quantum mechanical degeneracy pressure [9]. In the absence of proton decay, these remnants would live almost forever $[1,10]$. In this case, black holes would evaporate almost instantaneously compared to white dwarfs and neutron stars.

Since degenerate stars are far too extended to have event horizons, they cannot emit Hawking radiation in the usual manner available to black holes [11] (see also [6, 8, 12, 13]). Ref. [11] proved that in a globally static 
spacetime, such as that produced by a degenerate star, there is no particle creation. These results break down in black hole spacetimes because they are not globally static, where static means both time independent and invariant under time reversal. For black holes, the staticity breaks down at and beyond the event horizon.

After cooling to essentially zero temperature, degenerate stars can only lose energy through processes that utilize black holes, which can radiate. This avenue is studied in this present paper. Notice that this approach implicitly includes energy loss from proton decay via gravitation [14-17], since such processes involve microscopic black holes.

Motivated by the above discussion, we write the wave function of a cold degenerate star in the suggestive form

$$
|\operatorname{star}\rangle=\cos \theta\left|N_{\star}\right\rangle+\sin \theta|B H\rangle
$$

where $\left|N_{\star}\right\rangle$ represents the usual stellar configurations (superpositions of quantum mechanical states of $N_{\star}=$ few $\times 10^{57}$ particles) and $|B H\rangle$ represents superpositions of the possible black hole states. The total probability of the star being in a black hole state or containing a black hole is thus $\mathcal{P}=\sin ^{2} \theta \approx$ $\theta^{2} \ll 1$.

In general, the black hole part of the wave function will contain many different contributions, i.e.,

$$
|B H\rangle=\sum_{j} A_{j}\left|M_{\mathrm{bh}}\right\rangle
$$

where $\left|M_{\mathrm{bh}}\right\rangle$ represents the wave function for a black hole of mass $M_{\mathrm{bh}}$ and must be a superposition of many states. The quantity $\left|A_{j}\right|^{2}$ is the corresponding probability of the star being in a black hole state. The total probability is thus given by

$$
\mathcal{P}=\sin ^{2} \theta=\sum_{j}\left|A_{j}\right|^{2}
$$


We can estimate the probabilities $P_{j}=\left|A_{j}\right|^{2}$ from several different mechanisms, as discussed below.

\section{BLACK HOLE PROCESSES IN STARS}

\section{A. Dyson Tunneling}

On sufficiently long time scales, white dwarfs and neutron stars can experience tunneling events in which the star, or portions of the star, tunnel through the potential energy barrier produced by degeneracy pressure [10]. This process can, in principle, result in the formation of black holes.

We first consider the probability of tunneling through the barrier provided by degeneracy pressure. Such tunneling is a necessary but not sufficient condition for black hole formation. The time scales for tunneling processes are given by the standard formula,

$$
\tau=\tau_{0} \mathrm{e}^{S_{T}}
$$

where $\tau_{0}$ is the natural oscillation time of the system. The action integral $S_{T}$ can be written

$$
S_{T}=\frac{2}{\hbar} \int[2 M V(r)]^{1 / 2} d r
$$

where $V(r)$ represents the height of the potential energy barrier as a function of the radial coordinate $r$.

For a white dwarf supported by degenerate electrons, the potential energy can be obtained from integrals over the distribution functions for fermions [7]. Following standard formalism, we define $x=p_{F} / m_{e}$, where $p_{F}$ is the Fermi momentum and $m_{e}$ is the electron mass. The number density of electrons is then given by $n_{e}=x^{3} m_{e}^{3} / 3 \pi^{2}$ (we take $\hbar=1=c$ ). We want to consider a small spherical volume, of radius $r$, within the star that contains $N$ electrons and $(A / Z) N$ nucleons. After some algebra, the potential energy $V_{\mathrm{d}}[r(x)]$ due 
to degeneracy pressure can be written in the form

$V_{\mathrm{d}}=N m_{e} \frac{3}{8 x^{3}}\left[\left\{x\left(1+x^{2}\right)^{1 / 2}\left(1+2 x^{2}\right)-\ln \left[x+\left(1+x^{2}\right)^{1 / 2}\right]\right\}-1\right] \equiv N m_{e} \Phi(x)$,

where the second equality defines a dimensionless function $\Phi(x)$. In the limit of small $x$ (low density), this potential has the usual form associated with nonrelativistic degenerate fermions, i.e., $\Phi \propto x^{2} \propto r^{-2}$. In the opposite (relativistic) limit of large $x, \Phi \propto x \propto r^{-1}$. We also must include an additional contribution to the potential energy due to gravity. This potential can be represented with the approximate form

$$
V_{\mathrm{g}} \approx-f \frac{G M_{N}^{2}}{r-r_{S}}
$$

where $M_{N}=A N m_{P}$ is the total mass of the volume, $r_{S}=2 G M_{N}$ is the corresponding Schwarzschild radius, and we have defined a geometrical factor $f \approx 3 / 5$. Unless the radius $r$ approaches the Schwarzschild radius $r_{S}$, the degeneracy part of the potential completely dominates that of gravity. As a result, to a good approximation, we only need to include $V_{\mathrm{d}}$ in the action integral [6], i.e.,

$$
S_{T} \approx 2(9 \pi / 4)^{1 / 3}\left[2(A / Z) m_{P} / m_{e}\right]^{1 / 2} N^{4 / 3} \int_{x_{0}}^{\Omega} \frac{d x}{x^{2}} \Phi^{1 / 2}(x) .
$$

The integration ranges from starting values $x_{0}$ near unity, corresponding to the initial density of the volume, to a large upper cutoff $\Omega \gg 1$, corresponding to black-hole-like densities. The exact value of the upper cutoff does not matter in practice because the integral has almost all of its support for small $x$ (low density).

For white dwarfs, with $A / Z \approx 2$, we have

$$
S_{T} \approx 335 N^{4 / 3} I\left(x_{0}\right),
$$

where $I\left(x_{0}\right)$ is the integral evaluated for a given starting value of $x_{0}$, i.e., a given starting density. The fastest tunneling rate will occur in the center of 
the star where the density (and $x_{0}$ ) is maximal. For a typical white dwarf of mass $0.5 M_{\odot}$, the central density is about $10^{6} \mathrm{~g} / \mathrm{cm}^{3}, x_{0} \approx 0.80$, and $I\left(x_{0}\right) \approx$ 1.56. For a more massive white dwarf of $1.0 M_{\odot}$, the central density is about $3 \times 10^{7} \mathrm{~g} / \mathrm{cm}^{3}, x_{0} \approx 2.5$, and $I\left(x_{0}\right) \approx 1.0$.

For any given number $N$ of particles that are required to form a black hole, equation [10] determines the tunneling probability $\propto \exp \left[-S_{T}\right]$. We can thus estimate the probability (per natural oscillation time $\tau_{0}$ ) for white dwarfs to produce black holes through a tunneling process,

$$
P_{N}=\mathrm{e}^{-335 I\left(x_{0}\right) N^{4 / 3}},
$$

where $N$ determines the mass of the black hole $\left(M_{\mathrm{bh}} \approx(A / Z) N m_{P}\right)$. This process is exponentially suppressed as the number $N$ of particles increases, so the process is dominated by the minimum value of $N$.

As a reference point, if the minimum mass for a black hole is given by the Planck scale, $M_{\mathrm{pl}} \sim 2 \times 10^{-5} \mathrm{~g}$, we obtain $N \sim 10^{19}$ and hence $S_{T} \approx 10^{28}$. For this incredibly large value for the action, the natural vibration time scale $\tau_{0}$ becomes completely irrelevant.

The fastest possible rate for this process would be for the case $N=2$, and the action would be $S_{T} \approx 844 I\left(x_{0}\right)$. In the center of a relatively massive white dwarf, where $I \sim 1$, the corresponding time scale is $\tau \sim \mathrm{e}^{844} m_{P}^{-1} \sim$ $10^{336} \mathrm{yr}$. This time scale is still long compared to that of gravitationally induced proton decay $[1,14-16,18]$. We thus conclude that the tunneling of aggregates of baryons into black holes will not be an important process within white dwarfs.

The result is similar for the case of neutron stars [10]. In this case, we must replace the electron mass $m_{e}$ with the nucleon mass in all of the formulae and use $A / Z=1$. The resulting action is much smaller,

$$
S_{T} \approx 5.4 N^{4 / 3} I\left(x_{0}\right)
$$

At the center of a neutron star, the density is $\sim 10^{15} \mathrm{~g} / \mathrm{cm}^{3}$ and hence 
approaches nuclear denisty. As a result, $x_{0} \approx 1$, and hence $I\left(x_{0}\right) \approx 1.4$. The action then becomes $S_{T} \approx 7.6 N^{4 / 3}$. For the fastest case of only two nucleons, $N=2$, we have $S_{T} \approx 19$. This small action would seemingly imply a reasonably high probability of tunneling into a black hole. However, this calculation only takes into account the tunneling of neutrons through the potential barrier provided by their degeneracy pressure. For small values of $N$ and high densities, the neutrons start to overlap, and the quark structure of the nucleons must also be taken into account. The process of small numbers of quarks tunneling into a black hole is one allowed channel for gravitationally

induced proton decay and the estimated time scale is about $10^{45} \mathrm{yr}$ (see [14, 18] and also the following discussion).

\section{B. Kinematic Stellar Model}

In this approximation, we consider the star to be a collection of particles of mass $m$ with a number density $n$. We want to calculate the probability that $N$ particles happen to lie within their own Schwarzschild radius, i.e., that of a black hole of mass $M_{\mathrm{bh}}=m N$. In natural units, the volume of the hypothetical black hole is given by

$$
V_{\mathrm{bh}}=8 m^{3} N^{3} M_{\mathrm{pl}}{ }^{-6} .
$$

These $N$ particles generally occupy a much larger volume $V_{0}$ given by

$$
V_{0}=N / n
$$

Thus, the probability $p_{1}$ that a single particle lies within the black hole volume is simply

$$
p_{1}=\frac{V_{\mathrm{bh}}}{V_{0}}=8 n m^{3} N^{2} M_{\mathrm{pl}}{ }^{-6},
$$

and the probability $p_{N}$ that $N$ particles lie within the black hole volume is

$$
p_{N}=\left(p_{1}\right)^{N}=\left(8 n m^{3} N^{2} M_{\mathrm{pl}}^{-6}\right)^{N} .
$$


Since the star contains $N_{\star} / N$ volumes of $N$ particles, the total probability $P_{N}$ that the star contains a black hole composed of $N$ particles is given by

$$
P_{N}=N_{\star} N^{-1}\left(8 n m^{3} N^{2} M_{\mathrm{pl}}^{-6}\right)^{N}=N_{\star} \lambda^{N} N^{2 N-1}
$$

In the second equality, we have defined $\lambda=8 \mathrm{~nm}^{3} \mathrm{M}_{\mathrm{pl}}{ }^{-6} \approx 10^{-115} \ll 1$, where the numerical value assumes a nuclear density to evaluate $n$. For a white dwarf density, $n$ will be far smaller, and the value of $\lambda$ will decrease accordingly. For all values of $N$ less than $N_{*}$, the probability is a decreasing function of $N$. In other words, the most probable black hole to be formed kinematically will have the smallest possible value of $N$.

For the case in which the minimum black hole mass is the Planck mass, $N \approx 6 \times 10^{18}$, and hence $-\ln \left[P_{N}\right] \sim 10^{21}$. Loosely speaking, this time scale is somewhat longer than, but comparable to, the tunneling time scale calculated previously. On the other hand, for small values of $N$, the time scale is $\mathcal{O}\left(\lambda^{-1} n^{1 / 3}\right) \sim 10^{89}$ yr. For small values of $N$, one can also construct a kinematic model of the proton as a system of $N=3$ quarks and make a similar calculation to model proton decay [18].

\section{Virtual Black Holes}

In any quantum theory, one expects to find vacuum fluctuations associated with the fundamental excitations of the theory. Thus, in electrodynamics, one has the possibility of forming electron-positron pairs for a short time directly out of the vacuum. The existence of such processes can be observed indirectly by many quantum phenomena, such as the Casmir effect. However, this example illustrates only the simplest such possibility. One must also include in the vacuum processes all excitations of the theory. One should thus include the possibility of the production of proton-antiproton pairs, or even monopole-antimonopole pairs. These processes will generally be highly suppressed relative to the electron-positron amplitudes by virtue of their correspondingly large masses. 
In gravitation, one therefore expects not only to find virtual gravitons playing a role, but also virtual black holes. Unfortunately, however, the theory of gravitation is unrenormalizable. Although this difficulty prevents one from doing reliable calculations, but it is not unreasonable to suppose that a semi-classical calculation will give reasonable answers.

Einstein gravity is controlled by the action [19]

$$
I[g]=-\frac{1}{16 \pi G} \int_{M} R(-g)^{1 / 2} d^{4} x-\frac{1}{8 \pi G} \int_{\partial M} K(-h)^{1 / 2} C[h] d^{3} x,
$$

where $G$ is Newton's constant and $R$ is the Ricci scalar for the metric $g_{a b}$, which is defined on the spacetime $M$. The spacetime boundary $\partial M$ has the induced metric $h_{a b}$. The quantity $K$ is the trace of the second fundamental form on the boundary $\partial M$ and $C[h]$ is a functional of $h$ defined so that the action of Minkowski space vanishes. Extremization of this action for fixed metric on the boundary leads to the Einstein equations for $g_{a b}$ in $M$.

The path integral for gravity is

$$
Z \sim \int \mathcal{D}[g] \mathrm{e}^{i I[g]}
$$

where the integral is taken over all metrics $g$. Our goal is to investigate how black holes contribute to any amplitude in quantum gravity. We first assume that this integral can be approximated by the usual Euclidean continuation $[6,13,19]$. The action for a single black hole of mass $m$ is then given by

$$
I_{1}=\frac{4 \pi m^{2}}{M_{\mathrm{pl}}^{2}},
$$

where $M_{\mathrm{pl}}$ is the Planck mass. Ignoring interactions between the black holes, we find the action for a collection of $N$ black holes to be

$$
I_{N}=\frac{4 \pi N m^{2}}{M_{\mathrm{pl}}^{2}} .
$$

In the path integral, the black holes are indistinguishable; each is independent of the others and can be positioned anywhere in space. Since $N$ is 
undetermined, we can evaluate $Z$ in a box of volume $V$ to obtain the result

$$
Z \sim \int_{0}^{\infty} d m \sum_{N=0}^{\infty} \exp \left[-4 \pi N m^{2} / M_{\mathrm{pl}}^{2}\right] \frac{1}{N !}\left[\frac{V}{\ell_{\mathrm{pl}}^{3}}\right]^{N} .
$$

The factor of $V$ comes from accounting for the black holes being anywhere in the box, and the factor of $1 / N$ ! arises from their indistinguishability.

The combination of these results thus defines a probability distribution for having $N$ black holes with mass $m$. Elementary calculations yield the corresponding expectation values for the number density of black holes and for the black hole mass, i.e.,

$$
\langle n\rangle \sim \frac{V}{\ell_{\mathrm{pl}}^{3}} \quad \text { and } \quad\langle m\rangle \sim M_{\mathrm{pl}},
$$

where $\ell_{\mathrm{pl}}$ is the Planck length. Thus, spacetime must be filled with tiny Planck mass black holes with a density of roughly one per Planck volume. These microscopic virtual black holes will live roughly for one Planck time. This picture of the spacetime vacuum is sometimes called the spacetime foam.

Since black holes do not conserve baryon number, these virtual black holes contribute to the rate of proton decay due to the gravitational interaction. A proton can be considered to be a hollow sphere of radius $10^{-13} \mathrm{~cm}$ that contains three (valence) quarks. Suppose that two of these quarks fall into the same black hole at the same time. Since the black hole will decay predominantly into the lightest particles consistent with the conservation of charge and angular momentum, this process effectively converts the quarks into lighter particles. These particles will usually be electrons and neutrinos and hence baryon number conservation is generally violated. In other words, quantum gravity introduces an effective interaction leading to processes of the form

$$
q+q \rightarrow \ell+\nu
$$

This interaction can be regarding as a four-Fermi interaction whose coupling strength is determined by the Planck mass. This process is mediated by black 
holes and can violate conservation of baryon number. (Note that this process cannot be mediated by just gravitons because such interactions conserve both electric charge and baryon number.)

The probability of two quarks being within one Planck length $\left(\ell_{\mathrm{pl}} \sim\right.$ $\left.10^{-33} \mathrm{~cm}\right)$ of each other inside a proton is about $\left(m_{P} / M_{\mathrm{pl}}\right)^{3} \sim 10^{-57}$. This value represents the probability per proton crossing time $\tau_{P} \sim m_{P}^{-1} \sim 10^{-31}$ yr, if we assume that the particles move at the speed of light. In order for an interaction to take place (such as [24), a virtual black holes must be present at the same time that the two quarks are sufficiently near each other. Including this effect reduces the overall interaction probability by an additional factor of $m_{P} / M_{\mathrm{pl}}$. Converting these results into a time scale for proton decay, we find an estimated proton lifetime of $\tau_{P} \sim 10^{45} \mathrm{yr}$. Not surprisingly, this lifetime is also what one would expect from equation [1] if the unification mass $M_{X}$ is taken to be the Planck mass $M_{\mathrm{pl}}$.

\section{Time Scales for Evaporation vs Accretion}

Once a black hole exists within a star, two very different fates are possible. If the black hole is large enough, it will live long enough that it accretes additional material from the star before evaporating away. In this case, the black hole can eventually accrete the entire star. On the other hand, if the black hole is sufficiently small, it will evaporate before interacting with the stellar material. In this case, the legacy of the black hole is to leave behind its Hawking radiation products. Since black holes are known to not conserve baryon number in their evaporation processes $[6,8,13]$, the net result of this latter process is a mechanism for baryon decay.

In order to determine the mass of a black hole required to survive, rather than evaporate, we set the Hawking evaporation time equal to the interaction time (both time scales depend on the mass $M_{\mathrm{bh}}$ of the black hole). The evaporation time is given by

$$
\tau_{\text {evap }}=\tau_{\mathrm{pl}}\left(M_{\mathrm{bh}} / M_{\mathrm{pl}}\right)^{3} \text {, }
$$


where $\tau_{\mathrm{pl}}$ is the Planck time. The interaction time is given by

$$
\tau_{\mathrm{int}}=(n \sigma v)^{-1}=\frac{\tau_{\mathrm{pl}}}{4 \pi}\left(M_{\mathrm{bh}} / M_{\mathrm{pl}}\right)^{-2}\left(M_{\mathrm{pl}} / \Lambda\right)^{3},
$$

where we have introduced an energy scale $\Lambda$ which defines the number density of the star through the relation $n=\Lambda^{3}$ (notice that $\Lambda \sim 200 \mathrm{MeV}$ for a neutron star and $\Lambda \sim 0.1 \mathrm{MeV}$ for a white dwarf). Notice also we have taken $v=c=1$, which results in the shortest possible interaction time. The critical mass black hole, the smallest black hole that will survive rather than evaporate, is thus given by

$$
M_{\mathrm{bh}} *=M_{\mathrm{pl}}(4 \pi)^{-1 / 5}\left(M_{\mathrm{pl}} / \Lambda\right)^{3 / 5}
$$

For nuclear densities, such as those encountered in a neutron star, the critical mass black hole is about $M_{\mathrm{bh}} *=4 \times 10^{11} M_{\mathrm{pl}} \approx 4 \times 10^{6} \mathrm{~g}$ (about the mass of a tractor trailer). For the density of a white dwarf, the critical mass is somewhat larger, $M_{\mathrm{bh}} *=4 \times 10^{13} M_{\mathrm{pl}}$.

The corresponding values of $N$, the number of particles required to make the black hole, are also large: $N \approx 4 \times 10^{30}$ for the neutron star case and $N \approx 4 \times 10^{32}$ for the white dwarf.

All of the mechanisms for black hole formation favor small black holes over large ones. In particular, the most probable black holes will be much smaller than those required to survive and accrete the entire star, rather than evaporate. Even if the minimum possible mass for a black hole is as large as the Planck mass $M_{\mathrm{pl}}$, almost all black holes will still evaporate long before they can interact and grow. The net result of black hole processes within degenerate stars is thus to create a channel for the evaporation of mass energy. In other words, black hole processes lead to the decay of baryons and the loss of energy from the star. 


\section{LONG TERM STELLAR EVOLUTION}

Given the results obtained above, the long term evolution of cold degenerate stars is now clear: They will slowly evaporate.

The rate at which the stars evaporate depends on the black hole formation rate, which in turn depends on the minimum mass black hole that can form. If the smallest black hole mass is less than or comparable to the proton mass, then the net result will be gravitationally driven proton decay at a rate $\Gamma_{P}$. If the minimum black hole mass is larger and contains the mass equivalent of $N$ nucleons (e.g., if the Planck mass is the minimum black hole mass, $N$ $\approx 10^{19}$ ), then we can still define an effective nucleon decay rate through the relation

$$
\Gamma_{P}=N \Gamma_{\mathrm{bh}},
$$

where $\Gamma_{P}$ is the rate of black hole production. This form does not apply when the black hole mass $M_{\mathrm{bh}}=N m_{P}$ is larger than the critical mass $M_{\mathrm{bh}}$ * required to survive rather than evaporate (see equation [27]).

Once the decay rate for its constituent nucleons is determined, the star will evolve is a simple manner $[1,3]$. The total luminosity is given by

$$
L_{\star}=\Gamma_{P} M_{\star}(t)=\Gamma_{P} M_{\star 0} \mathrm{e}^{-\Gamma_{P} t},
$$

where $M_{\star}$ is the stellar mass and $M_{\star 0}$ is its initial mass.

The decay products from black hole evaporation can be divided into two general classes: those that interact with stellar material before leaving the star (optically thick components) and those that leave with out interacting (optically thin components). Photons and all charged particles will be optically thick, whereas neutrinos and gravitons will be optically thin. The fraction $\mathcal{F}$ of optically thick components will generally thermalize within the stellar interior and ultimately produce a photon luminosity

$$
L_{\star \gamma}=\mathcal{F} L_{\star}=4 \pi R_{\star}^{2} \sigma_{B} T_{\star}^{4}
$$


In the second equality we have defined the corresponding surface temperature of the star through the usual black body relation, where $\sigma_{B}$ is the StefanBoltzmann constant. The stellar radius $R_{\star}$ is determined by the usual massradius relation for cold degenerate stars [7, 9],

$$
R_{\star}=1.42\left(\frac{M_{\mathrm{pl}}}{m_{D}}\right)\left(\frac{M_{\mathrm{pl}}}{m_{P}}\right)\left(\frac{M_{\star}}{m_{P}}\right)^{-1 / 3} m_{P}^{-1},
$$

where $m_{D}$ is the mass of the particle responsible for the degeneracy pressure, i.e., $m_{D}$ is the electron mass $m_{e}$ for white dwarfs and the neutron mass $m_{N}$ for neutron stars.

Combining equations [30] and [31], we obtain an expression for the evolutionary tracks of the stars in the Hertzsprung-Russell (H-R) diagram,

$$
L_{\star \gamma} \approx 1.5\left(4 \pi \sigma_{B}\right)^{3 / 5}\left(\mathcal{F} \Gamma_{P}\right)^{2 / 5}\left(\frac{M_{\mathrm{pl}}^{2}}{m_{D} m_{P}}\right)^{6 / 5}\left(\frac{M_{\star}}{m_{P}}\right)^{-2 / 5} m_{P}^{-4 / 5} T_{\star}^{12 / 5} .
$$

In this regime, stellar evolution is thus determined once the effective nucleon decay rate $\Gamma_{P}$ is specified. Unfortunately, as discussed above, the decay rate $\Gamma_{P}$ can take a rather wide range of values. The resulting evolution is shown in Figure 1 for the representative value of $\Gamma_{P}=\left(10^{45} \mathrm{yr}\right)^{-1}$.

Degenerate objects become larger in radial size as they lose mass. White dwarfs will follow the evolutionary tracks given by equation [32] until they expand to a size for which they are no longer degenerate. At this point in the star's future evolution, when the stellar mass has fallen to $\sim 10^{-3}$ $M_{\odot}$, the star's track in the H-R diagram will change its slope. The above expressions for the stellar luminosity and the surface temperature remain valid, but the mass-radius relation [31] no longer applies. The structure of non-degenerate matter is largely determined by Coulomb forces, which enforce roughly uniform density $\rho_{0}$, and the star follows an evolutionary track [1] given by

$$
L_{\star}=\frac{36 \pi \sigma_{B}^{3}}{\mathcal{F}^{2} \Gamma_{P}^{2} \rho_{0}^{2}} T_{\star}^{12}
$$


where $\rho_{0} \sim 1 \mathrm{~g} / \mathrm{cm}^{3}$. At the start of this phase of evolution, the object has a mass, radius, and density closely akin to those of the planet Jupiter. The object then continues to lose its mass until it can no longer be considered a star. The end point of stellar evolution occurs when the mass has fallen to about $10^{24} \mathrm{~g}$, the point where the object can no longer completely thermalize its internal radiation.

Neutron stars follow a parallel evolutionary track given by equation [32], where $m_{D}$ is now the neutron mass. However, as they lose mass and the neutrons are lifted out of degeneracy, neutron stars must eventually experience a significant readjustment when the mass drops below $\sim 0.0925 M_{\odot}$ [7]. During this readjustment phase, a neutron star will either expand to a white dwarf configuration [1] or explode and widely disperse its remaining mass [20]. These possibilities are illustrated by the dashed curve and arrows shown in Figure 1.

For completeness and comparison, we note that stellar black holes evolve in a completely different manner. As black holes lose mass through Hawking evaporation, they become smaller while their surface temperature and luminosity increase. Black holes follow an unambiguous track in the H-R diagram given by

$$
L_{\mathrm{bh}}=\frac{\sigma_{B}}{4 \pi} T_{\mathrm{bh}}^{2} .
$$

A portion of the black hole evolutionary path is shown in the lower left part of Figure 1. This evolution continues until the black hole mass dwindles to the Planck mass and the surface temperature reaches the Planck temperature. Beyond this critical juncture, further black hole evolution depends on quantum gravity effects and is largely unknown.

Notice that the long term evolution of white dwarfs is completely specified by equations [32] and [33], and that all of the parameters are known except for the value of the effective proton decay rate $\Gamma_{P}$. For black holes, the long term evolution is given by equation 34 with absolutely no unknown 
parameters.

\section{SUMMARY}

The final demise of white dwarfs and neutron stars will take place on a time scale that vastly exceeds the current age of the universe. However, in the absence of conventional unification scale proton decay, the black hole processes outlined here will determine the ultimate fate of these cold degenerate stellar remnants. Within the stars, the formation of small black holes is strongly favored over large black holes and hence essentially all processes result in black holes that evaporate rather than accrete additional stellar material. The net result is that degenerate stars will themselves evaporate and follow the evolutionary tracks depicted in Figure 1. Since the vast majority of stars are destined to end their conventional nuclear burning lives as white dwarfs, with most of the remainder becoming neutron stars, this work describes the long term fate and evolution of almost all of the stars in the sky.

\section{Acknowledgements}

We would like to thank G. Kane for facilitating the visit of M. Perry to U. Michigan. We also thank A. Zytkow for interesting discussions. This work was supported by a Department of Energy grant, an NSF Young Investiga-

tor Award, NASA Grant No. NAG 5-2869, and by funds from the Physics Department at the University of Michigan. 


\section{REFERENCES}

[1] F. C. Adams and G. Laughlin, Rev. Mod. Phys. 69, 337 (1997).

[2] P. Langacker, Phys. Rep. 72, 186 (1984).

[3] D. A. Dicus, J. R. Letaw, D. C. Teplitz, and V. L. Teplitz, ApJ, 252, 1 (1982).

[4] G. Feinberg, Phys. Rev. D 23, 3075 (1981).

[5] S. W. Hawking, Comm. Math. Phys. 43, 199 (1975); S. W. Hawking, Nature 248, 30 (1974).

[6] N. D. Birrell and P. C. W. Davies, Quantum Fields in Curved Space (Cambridge Univ. Press, Cambridge, 1982).

[7] S. L. Shapiro and S. A. Teukolsky, Black Holes, White Dwarfs, and Neutron Stars (Wiley, New York, 1983).

[8] K. S. Thorne, R. H. Price, and D. A. MacDonald, Black Holes: The Membrane Paradigm (Yale Univ. Press, New Haven, 1986).

[9] S. Chandrasekhar, Stellar Structure (Dover, New York, 1939).

[10] F. J. Dyson, Rev. Mod. Phys. 51, 447 (1979).

[11] J. Dimock and B. S. Kay, Annales Inst. H. Poincare, A 37, 93 (1982); J. Dimock and B. S. Kay, Annals Phys. 175, 366 (1987).

[12] I. D. Novikov and V. P. Frolov, Physics of Black Holes (Kluwer, Dordrecht, 1989).

[13] R. M. Wald, Quantum Field Theory in Curved Spacetime and Black Hole Thermodynamics (Univ. Chicago Press, Chicago, 1994).

[14] Ya. B. Zeldovich, Phys. Lett. 59 A, 254 (1976); Ya. B. Zeldovich, Sov. Phys. JETP, 45, 9 (1977). 
[15] S. W. Hawking, D. N. Page, and C. N. Pope, Phys. Lett. 86 B, 175 (1979).

[16] D. N. Page, Phys. Lett. 95 B, 244 (1980).

[17] J. Ellis, J. S. Hagelin, D. V. Nanopoulos, and K. Tamvakis, Phys. Lett. 124 B, 484 (1983).

[18] M. J. Perry, in Unification of Elementary Forces and Gauge Theories, ed. D. B. Cline and F. E. Mills, p. 485 (Harwood, London, 1977).

[19] G. W. Gibbons and S. W. Hawking, Phys. Rev. D 15, 2752 (1977).

[20] D. N. Page, Phys. Lett. 91 A, 201 (1982).

\section{Figure Caption}

Figure 1. The long term evolution of cold degenerate stars in the H-R dia-

gram. Upon completing the early stages of stellar evolution, white dwarfs and neutron stars rapidly cool to an equilibrium temperature dictated by black hole induced proton decay (assumed here to occur at rate $\Gamma_{P}=\left[10^{45} \mathrm{yr}\right]^{-1}$ ). The white dwarf models are plotted at successive twofold decrements in mass. The mean stellar density (in $\log [\rho / \mathrm{g}]$ ) is indicated by the gray scale shading, and the sizes of the circles are proportional to stellar radius. The relative size of the Earth and its position on the diagram are shown for comparison. The evaporation of a $1 M_{\odot}$ neutron star is illustrated by the parallel sequence, which shows the apparent radial sizes greatly magnified for clarity. The Hawking radiation sequence for black holes is also plotted. The arrows indicate the direction of time evolution. 


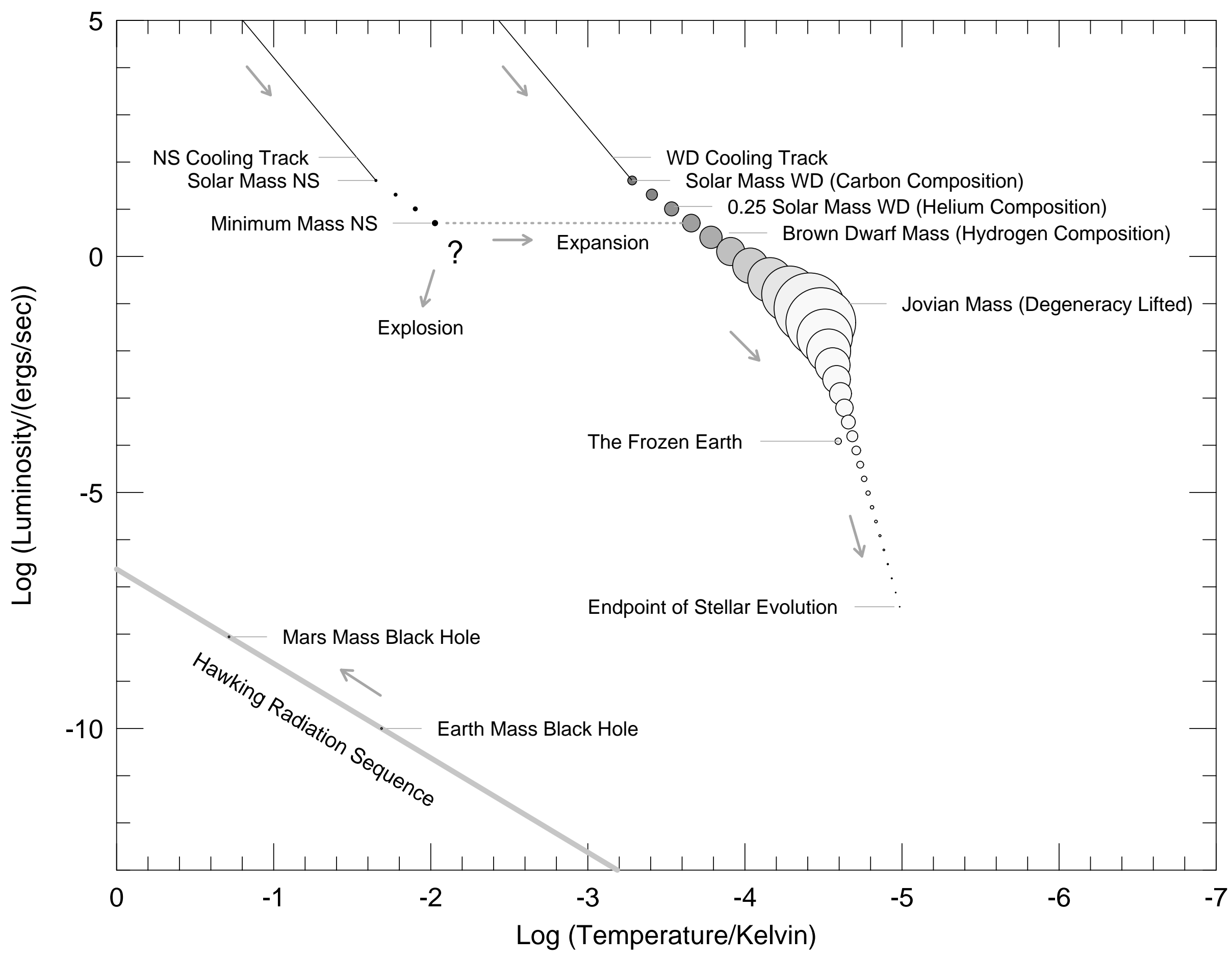

